\title{
Posture Stability Evaluation Using Static Posturography in Patients after Cruciate Ligament Reconstruction
}

\author{
L. Kubisz ${ }^{a, b, *}$, H. Werner ${ }^{a}$, M. BoseK ${ }^{c}$ And W. Weiss ${ }^{d}$ \\ ${ }^{a}$ Department of Biophysics, Poznań University of Medical Sciences, Poznań, Poland \\ ${ }^{b}$ Higher Vocational State School, Piła, Poland \\ ${ }^{c}$ Department of Biophysics, Collegium Medicum in Bydgoszcz, Nicolaus Copernicus University, Poland \\ ${ }^{d}$ Orthopedic and Trauma Ward Centre for Complex Sport Injures Treatment \\ Collegium Medicum in Bydgoszcz, Nicolaus Copernicus University, Poland
}

\begin{abstract}
Ligament reconstruction is one of the surgical methods for treatment of anterior cruciate ligament injury. The main purpose of rehabilitation after surgical ligament reconstruction is to obtain maximal efficiency of knee joint, their stability and appropriate lower limbs encumbrance. Static posturography is one of the control, rehabilitative and diagnostic tools in such treatment. The purpose of the study was to evaluate the posture stability differences between healthy people and patients after anterior cruciate ligament reconstruction. The centre of pressure sway was registered using the standard posturography platform (Pro-med). During the test, the subject stood freely with eyes open and closed. In order to determine the stability of the subject, the average velocity of the centre of pressure sway, and the average accretion rate of surface area encircled by centre of pressure, a mean radius of centre of pressure and amount of its displacement were calculated. Parameters measured for the eyes closed posture were significantly greater for people after anterior cruciate ligament reconstruction, while differences between groups for eyes open test were statistically insignificant. This indicates impairment of the somatosensoric posture control system. It should be noted that selected stability indicators: an average velocity of the centre of pressure sway, its anterior-posterior and mediolateral components, a mean radius of centre of pressure and a mean accretion rate of surface area encircled by centre of pressure varied in both groups.
\end{abstract}

PACS: 87.19.lu, 87.19.R-, 87.19.U-

\section{Introduction}

Among all knee ligament injuries the most often found is the disruption of the anterior cruciate ligament (ACL). The ACL insufficiency causes anterior knee instability which, in time, may lead to gonarthrosis (knee joint degeneration). Usually young and physically fit people, are liable to sustain accidents, mostly due to inappropriate preparation. Several therapeutical methods have been developed over the years. Their primary objective is to obtain maximal joint efficiency - regeneration of muscle mass and strength, mobility range, joint stability and appropriate lower limbs encumbrance. One of the main functions of the ACL is to secure tibia bone from excessive translocation in relation to femur (tight bone) and a 10 degree rotation control of tibia in the final phase of extension. Cruciate ligament also limits extension and flexion range of the knee joint, as well as internal rotation of tibia and flexed knee frontal plane dislocation. Besides joint mechanics, the ACL is also responsible for somatosensory system of posture control [1-8].

Balance regulation in healthy people influences appropriate body position in rest, as well as in motion. Pos-

* corresponding author; e-mail: kubisz@amu.edu.pl tural stability is the ability to actively restore a typical for human body position when disturbed by various factors [9]. People may lose balance due to imprecise movements without considering all mechanic constituents or occurrences of unpredictable external factors such as obstacles and slippery surfaces $[10,11]$. Certain factors may impair the efficiency of the balance control system and mobility coordination. The most significant effect of the posture stability impairment is the mobility disorder. It is the core of the orthopedical and traumatological based afflictions [10].

The evaluations of the rehabilitation progress in patients after the ACL reconstruction are a result of many actions. In physical therapy the patient undergoes several functional or clinical tests. Muscle mass, strength and joint mobility range are assessed. Additionally, self-assessment questionnaires are used. For the purpose of proprioceptic control evaluation, a posturography platform can be used [12,13]. Posturography is a group of methods allowing the evaluation of the posture control $[14,15]$.

Studies on efficiency and accuracy of proprioception tests after the ACL reconstruction can be carried out by means of dynamic tests. Usually they are used after 6,12 and 36 months of rehabilitation $[8,16]$. The tests applied for the balance evaluation may use various destabilizing 
factors, like support surface modification (one foot standing), dynamic tests (jumping in place or using electronic platforms with infinite degrees of freedom). Stability control is also impaired by disruption of the sensory system involved in the balance support especially when visual stimuli are limited [11, 12, 17-19].

Static tests are another kind of posturographic examinations. Since centre of pressure (COP) for standing person permanently sways, a stabilograph of mediolateral and anterior-posterior sways can be registered as a function of time. Such examinations require quasi-static environment and may be performed at the early stage of rehabilitation. Furthermore, results of such tests can be used as a benchmark for dynamic tests at later stages of rehabilitation.

Since the post-procedure evaluation process had been introduced early, the study was carried out using static posturography. The purpose of the study was to determine differences between the group of patients after the ACL reconstruction and the group of healthy people.

\section{Material and methods}

The research was carried out on a test group of 10 young people after the ACL reconstruction and a control group of 17 people. Both groups consisted of people of similar ages ranging between 20 and 37, and similar body structures (1.63-1.96 m height, 50-94 kg body mass). All subjects were physically active. The test group was composed of patients six weeks after surgical ACL reconstruction. All patients were operated using the same procedure and rehabilitated according to a specific model designed to obtain full motion range, muscle strength and proprioception. None of the patients was diagnosed with neurological disorders affecting motoric or posture control.

The COP displacements were registered using a standard posturography platform Pro-med (Janusz Olton, Poland) connected to a computer. During measurements, the subject stood freely with eyes open and closed. A single measurement time was $36 \mathrm{~s}$, including 4 seconds position test, and a $30 \mathrm{~s}$ intervals. For the purpose of stability, the evaluation of examined persons, an average velocity of movement of the COP sway, an average accretion rate of surface area encircled by the COP, and a mean radius of the COP and amount of its displacement were used. The obtained values for both tests were compared between the two groups for eyes open and closed measurements. Collected data were analyzed using the ShapiroWilk test, the Mann-Withney test and the Wilcoxson signed rank test (Statistica software).

\section{Results}

Figure 1 and Fig. 2 present stabilograms and the COP displacements as a function of time for the test and the control groups during the quiet standing. Significantly higher values of displacements are seen for patients after the ACL reconstruction. Figures 3-7 present the
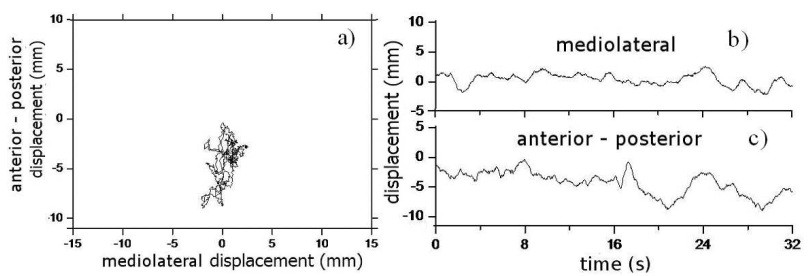

Fig. 1. Trajectory of the COP sway (a), mediolateral (b) and anterior-posterior (c) components of the COP sway, the control group.
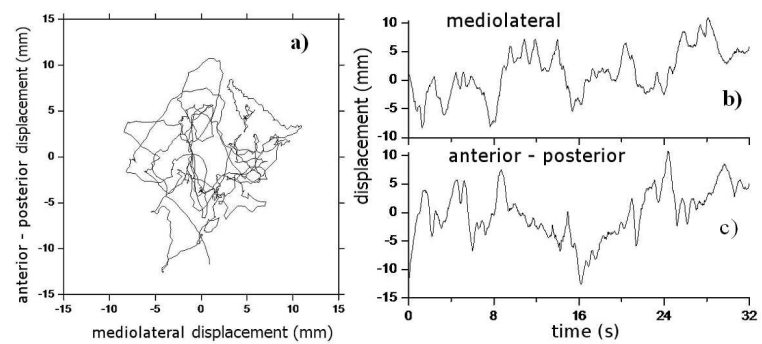

Fig. 2. Trajectory of the COP sway (a), mediolateral (b) and anterior-posterior (c) components of the COP sway, the test group.

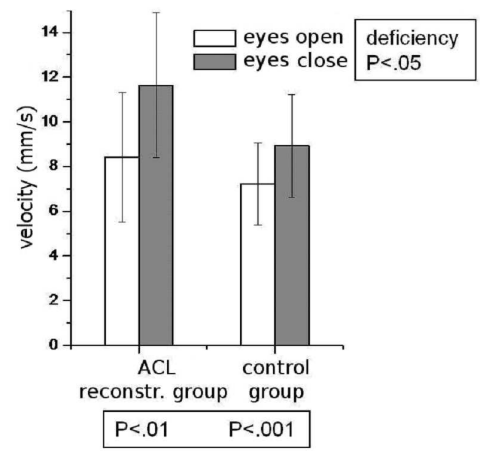

Fig. 3. Velocity of movement of the COP sway, the control and the test group (mean \pm standard deviation).

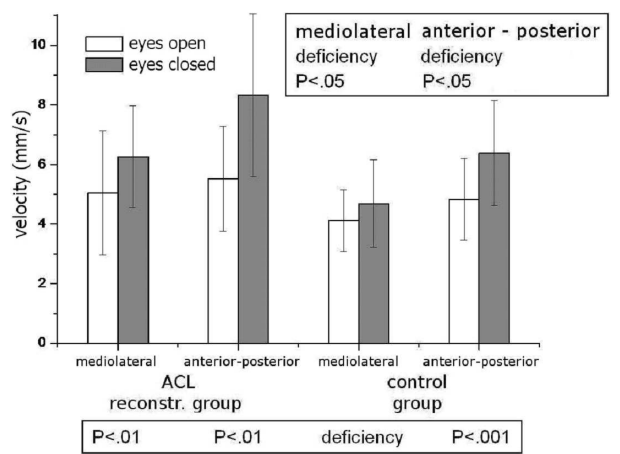

Fig. 4. Velocity of movement of the COP sway components, the control and the test group (mean \pm standard deviation). 


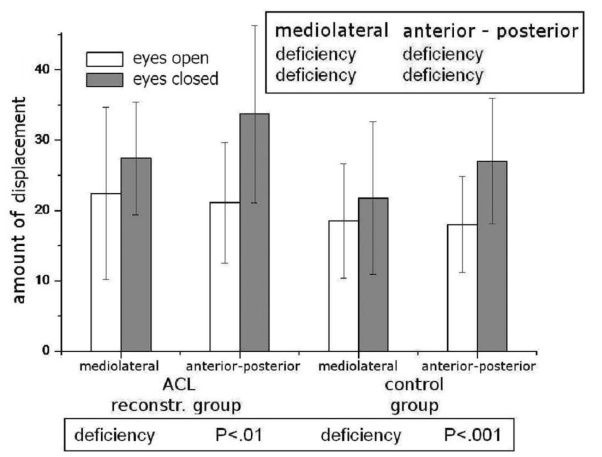

Fig. 5. Amount of the COP displacement, the control and the test group (mean \pm standard deviation).

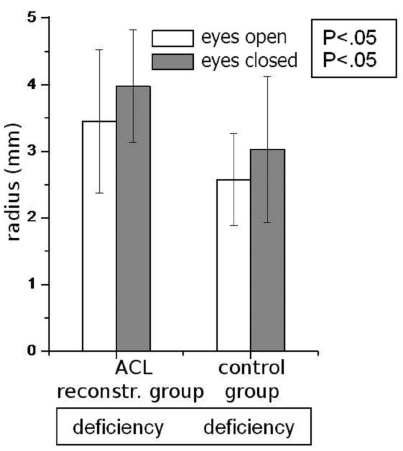

Fig. 6. The COP displacement radius, the control and the test group (mean \pm standard deviation).

means of the results, their standard deviations and results of statistical tests. While comparing the average velocity of movement of the COP sway and its two components (mediolateral and anterior-posterior) registered for eyes open test, the differences found were statistically insignificant. The same test carried out on people with eyes closed proved to obtain significantly higher values for patients after the ACL reconstruction. The differences in the velocity of movement of the COP sway and

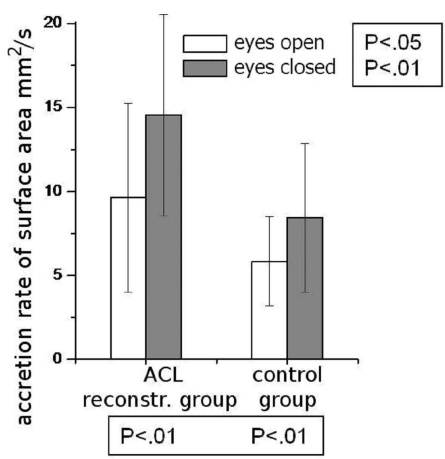

Fig. 7. Accretion rate of surface area encircled by the COP, the control and the test group (mean \pm standard deviation). its components in sagittal plane (anterior-posterior) between open and eyes closed tests were statistically significant among both groups. No significant differences were observed in the amount of the COP displacements in both coronal and sagittal planes for open, as well as closed eye test. Internal group comparisons between eyes open and closed tests found significant differences in values measured for the sagittal plane, but they were insignificant for the coronal plane. During tests under eyes open and closed, it was observed that the average values of COP displacement radius were significantly higher in the test group. Internal group comparisons found no significant results. Assessment of the average accretion rate of surface area encircled by the COP during experiments resulted in significantly higher values for people after the ACL reconstruction. Furthermore, when comparing results significant differences were found for tests with eyes open and closed.

\section{Discussion}

Results obtained in the study for selected factors proved to have higher values among people after the ACL reconstruction. This indicates impairment of the motor or somatosensory posture control systems. Pathological changes in knee joint in examined patients may influence degradation of mechanical receptors. On the other hand, analyzed parameters increased their values when tests were performed with eyes closed. This impairment was especially visible in anterior-posterior direction. Nevertheless, shutting off visual stimuli proved to have a much higher impact on the posture control system disorders in subjects after the ACL reconstruction. This indicates that the balance control impairment after the ACL reconstruction procedure is compensated with greater involvement of visual sensors.

The analysis of the results shows that the visual control system supports for mechanical receptors improving the posture control. This system works in conjunction with oscillating head movements. When standing freely with eyes open head movements are minimal. They increase in amplitude and frequency when eyes are closed [20].

Rehabilitation models are developed to limit the visual compensation and improve somatosensory components of the posture control system. Therefore, the treatment starts from activities performed with eyes open. Next, visual stimuli are consequently limited until completely shut down [13].

Dissimilar values of stability factors in the test group than in the control group may be influenced not only by proprioceptic senses. This condition might be also a result of quadriceps femoris muscle injury resulting from the ACL surgical procedure, where the part of this muscle is used for ligament reconstruction. Quadriceps actively stabilizes knee joint during extension. Quadriceps belong to a part of anti-gravity muscle group. Their permanent but low tension is necessary for keeping a balanced vertical body posture. One can find the presence of the 
correlation between the extension value of knee joint and probability of falling, which had been reported. There was described the relation between an under-knee angle value and the integrated muscle activity also for the quadriceps muscle. It was concluded that the maximal extension of the knee joint is one of the mechanisms influencing functional stability of joint and it reduces the probability of falling [20].

\section{Conclusions}

The obtained results proved that posturographic tests are appropriate for the posture evaluation in patients after ACL surgical reconstruction and for the determination of the rehabilitation efficiency. The selected COP roaming parameters are simple and efficient in describing the mechanisms used by the posture control system. Probably, static posturography is the method, which can be applied in the evaluation and comparison of different method of rehabilitation in the ACL injuries, especially such as magnetotherapy and magnetostimulation.

The additional conclusion is, that after six week rehabilitation after reconstruction of the ACL the somatosensoric posture control system was still damaged.

\section{References}

[1] A. Stolarczyk, R. Śmigielski, G. Adamczyk, Medycyna Sportowa 10, 23 (2000) (in Polish).

[2] D.J. Beard, P.J. Kyberd, M. Fergusson, C.A. Dodd, J. Bone Joint Surg. (Brit.) 75-B, 311 (1995).

[3] B.D. Beynnon, S.H. Ryder, L. Konradsen, R.J. Johnson, K. Johnson, P.A. Renstrom, Am. J. Sports Med. 27, 150 (1999)

[4] J. Jerosch, M. Prymka, Arch. Orthop. Trauma Surg. 115, 162 (1996).
[5] S.M. Lephart, M.S. Kocher, F.H. Fu, P.A. Borsa, C.D. Harner, J. Sports Rehab. 1, 188 (1992).

[6] B. Zouita, A. Moussa, S. Zouita, C. Dziri, F.Z. Ben Salah, Ann. Phys. Rehab. Med. 52, 475 (2009).

[7] H. Johansson, P. Sjölander, P. Sojka, Crit. Rev. Biomed. Eng. 18, 341 (1991).

[8] R. Zätterström, T. Fridén, A. Lindstrand, U. Moritz, Am. J. Sports Med. 22, 531 (1994).

[9] C.G. Danis, D.E. Krebs, K.M. Gill-Body, Phys. Ther. 78, 502 (1998)

[10] E. Szczepek, L. Czerwosz, P. Dabrowski, K. Dudziński, J. Jurkiewicz, Z. Czernicki, Neurologia i Neurochirurgia Polska 42, 139 (2008) (in Polish).

[11] J. Błaszczyk, Biomechanika kliniczna, PZWL, Warszawa 2004, p. 203, (in Polish).

[12] M. Dzierżanowski, G. Srokowski, W. Hagner, Fizjoterapia 11, 39 (2003) (in Polish).

[13] S.B. Brotzman, K.E. Wilk, A. Dziak, Rehabilitacja ortopedyczna 2, 399 (2008) (in Polish).

[14] M. Bosek, B. Grzegorzewski, A. Kowalczyk, Movement Sci. 22, 649 (2004).

[15] M. Bosek, B. Grzegorzewski, A. Kowalczyk, I. Lubiński, Neurosci. Lett. 16, 215 (2005).

[16] M. Henriksson, T. Ledin, L. Good, Am. J. Sports Med. 29, 359 (2001).

[17] J. Olton, L. Czerwisz, Acta Bio-Opt. Informat. Med. 12, 143 (2006)

[18] M. Shiraishi, H. Minuta, K. Kubota, Y. Otsuka, N. Nagamoto, K. Takagi, Clinical J. Sport Med. 6, 32 (1996).

[19] T.R. Bonfim, D.B. Grossi, C.A. Paccola, J.A. Barela, Neurosci. Lett. 29, 257 (2008).

[20] T. Piontek, K. Ciemniewska-Gorzela, A. Szulc, A. Pyda, W. Dudziński, R. Hejna, Chirurgia Narzadów Ruchu i Ortopedia Polska 74, 353 (2009) (in Polish). 